\title{
Phase mapping of ultrashort pulses in bimodal photonic structures: A window on local group velocity dispersion
}

\author{
H. Gersen, ${ }^{1, *}$ E. M. H. P. van Dijk, ${ }^{1}$ J. P. Korterik, ${ }^{1}$ N. F. van Hulst, ${ }^{1}$ and L. Kuipers ${ }^{1,2}$ \\ ${ }^{1}$ Department of Science \& Technology, Applied Optics Group, and $\mathrm{MESA}^{+}$Research Institute, University of Twente, \\ P.O. Box 217, 7500 AE Enschede, The Netherlands \\ ${ }^{2}$ FOM-Institute for Atomic and Molecular Physics (AMOLF), Kruislaan 407, 1098 SJ Amsterdam, The Netherlands
}

(Received 20 January 2004; published 8 December 2004)

\begin{abstract}
The amplitude and phase evolution of ultrashort pulses in a bimodal waveguide structure has been studied with a time-resolved photon scanning tunneling microscope (PSTM). When waveguide modes overlap in time intriguing phase patterns are observed. Phase singularities, arising from interference between different modes, are normally expected at equidistant intervals determined by the difference in effective index for the two modes. However, in the pulsed experiments the distance between individual singularities is found to change not only within one measurement frame, but even depends strongly on the reference time. To understand this observation it is necessary to take into account that the actual pulses generating the interference signal change shape upon propagation through a dispersive medium. This implies that the spatial distribution of phase singularities contains direct information on local dispersion characteristics. At the same time also the mode profiles, wave vectors, pulse lengths, and group velocities of all excited modes in the waveguide are directly measured. The combination of these parameters with an analytical model for the time-resolved PSTM measurements shows that the unique spatial phase information indeed gives a direct measure for the group velocity dispersion of individual modes. As a result interesting and useful effects, such as pulse compression, pulse spreading, and pulse reshaping become accessible in a local measurement.
\end{abstract}

DOI: $10.1103 /$ PhysRevE.70.066609

PACS number(s): 42.25.-p, 78.47.+p, 68.37.Uv, 42.65.Re

\section{INTRODUCTION}

Dispersion is the phenomenon that the refractive index of a medium varies with frequency [1]. Vacuum exhibits no dispersion, so that the phase velocity and the group velocity of a wave packet centered at frequency $\omega$ are both constant and equal to $c$. In all other optical media, the presence of resonances modifies this dispersion relation so that the phase and group velocities are different, even in regions far away from resonance [2]. An important consequence is that both velocities are functions of frequency, having the effect that a short optical pulse changes shape as it propagates $[2,3]$.

Besides the material properties, dispersion is also influenced by interference of waves in periodic and waveguiding structures. As the dispersion depends on the periodicity in the structure it can therefore be tuned over a wide range, including regions of negative dispersion [2]. Recently, photonic crystals ( $\mathrm{PhC}$ 's) have shown that the dispersion relation itself can be engineered in a unique fashion [4]. $\mathrm{PhC}$ 's are periodic optical structures on the length scale of the electromagnetic wavelength aimed at controlling the generation and propagation of light [5]. PhC's are extremely attractive for integrated optical circuits as different functionalities can simply be introduced by modifying the local symmetry and geometry of the $\mathrm{PhC}$ lattice [6]. This corresponds to a modification of the photon dispersion relation and ultimately leads to tailoring of group velocity dispersion (GVD), photonic band gaps, and localized states.

\footnotetext{
*Corresponding author. Present address: Center for Atomic-Scale Materials Physics, University of Aarhus, DK-8000 Århus C, Denmark. Electronic address: h.gersen@alumnus.utwente.nl
}

Direct determination of the GVD of an optical pulse propagating in a $\mathrm{PhC}$ device is crucial to the demonstration of useful dispersive properties of PhC devices. So far, many investigations on dynamical effects in (non)linear dispersive media are based on numerical simulations [7-10]. However, experimental verification of the dispersive properties of $\mathrm{PhC}$ 's is still a difficult and demanding issue. Several groups have indirectly extracted the dispersive properties from the phase of Fabry-Perot oscillations between a PhC section and sample facets (which are superimposed on emission spectra from their samples) [11-13]. Time-resolved experiments have been reported which give insight into the group velocity upon propagation through two-dimensional (2D) photonic crystal waveguides either by optical Kerr gating [14] or by a cross-correlation method [15]. These methods, however, integrate all the pulse propagation effects accumulated along the entire structure. If a structure has spatially varying optical properties, only averaged information is obtained. By cutting slices from the medium ("cut-back" method), it becomes possible to study aspects of the internal pulse development [16]; yet this method is destructive and it is obvious that a photonic structure as a $\mathrm{PhC}$ cannot be arbitrarily changed in length without affecting its properties. Furthermore, if a disagreement is found between experiment and theory it may be hard to find the underlying cause for the discrepancy. To overcome these drawbacks and obtain full local information on pulse propagation throughout a medium, local timeresolved measurements are crucial.

In the last years, near-field scanning optical microscopy (NSOM) has proven to be a powerful method to analyze local electromagnetic field distributions in fabricated nanophotonic structures [17-23]. It has been demonstrated that 
the combination of NSOM with coherent methods yields the amplitude and phase distribution of the optical field locally inside the structure [24-28]. Extension of this approach towards femtosecond pulses enables the observation of dynamical effects directly inside a photonic structure as we recently have demonstrated $[29,30]$.

Here, we report on the nondestructive visualization of pulses as they propagate through bi-modal photonic structures at infrared wavelengths. Unlike most measurements which reconstruct the group velocity from interferometric measurement, we directly measure the phase and group velocity for individual modes locally inside the waveguide. When the waveguide modes overlap in time, unexpected phase patterns are observed. Phase singularities were expected at equidistant intervals determined by the difference in effective index for the two modes. However, we observe that the distance between individual singularities not only changes within one measurement frame, but also depends strongly on the reference time. It is necessary to take into account the fact that the actual pulses generating the interference signal are no longer Fourier limited due to changes in shape resulting from propagation through the dispersive media. Consequently, it is expected that the location of phase singularities yields direct information on the local dispersion characteristics. In the following, we will show how the spatial distribution of phase singularities allows the retrieval of the GVD for the individual modes by means of an analytical model of the time-resolved photon scanning tunneling microscope (PSTM) measurements [30]. With this method propagation effects, such as pulse compression, pulse spreading, and pulse reshaping, become accessible in a local measurement.

\section{EXPERIMENTAL ASPECTS}

Near-field optics provides a powerful method to circumvent the diffraction limit [31]. Instead of imaging with a system of lenses, a glass fiber with a subwavelength sized end facet can be used to probe the light fields close to a sample surface. By making the distance between fiber probe and sample much smaller then the wavelength of light (typically $<10 \mathrm{~nm}$ ), nonpropagating light fields that are bound to the sample surface can be detected as schematically depicted in Fig. 1. These "evanescent" fields decay in magnitude within a fraction of the wavelength when moving away from the surface, and carry information about sample features smaller than the value set by the diffraction limit. Owing to their nonpropagating character, evanescent fields can only be detected by a local probe immersed into the near-field of the sample [32]. The evanescent field is locally converted into a propagating wave, which is coupled into the fiber probe, guided through the fiber and subsequently detected. The corresponding experimental device is called a near-field optical microscope in collection mode, hereafter called a photon scanning tunneling microscope (PSTM).

Interferometric detection of the optical signal from the fiber probe provides an additional source of information by providing access to both the amplitude and phase of the probed field $[24,27,28]$. The photon tunneling signal picked

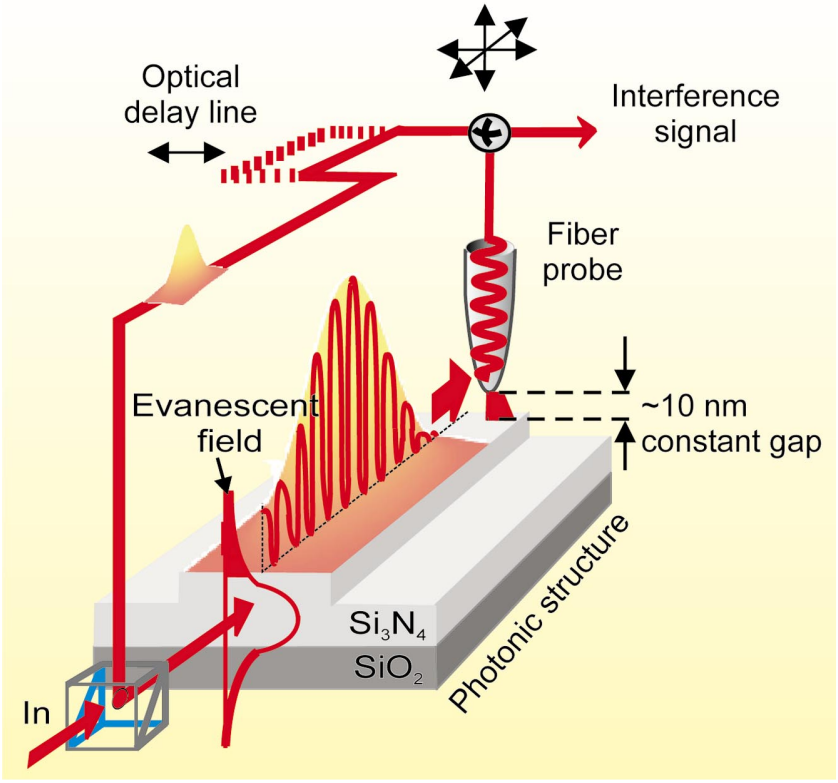

FIG. 1. (Color) Schematic representation of a pulse tracking experiment. The evanescent field of the pulse traveling inside a waveguide is picked up by a fiber probe with subwavelength dimensions. The photon tunneling signal picked up by the probe is interferometrically mixed with part of the same pulse that has propagated through the reference branch. The length of the reference branch, and thus the time that it takes the reference pulse to travel through this branch, is controlled by an optical delay line. Each subsequent measurement shown in this paper is obtained by raster scanning the optical probe across the photonic structure while the height above the structure $(<10 \mathrm{~nm})$ is kept constant by a feedback mechanism. The simultaneous measurement of optics and topography makes it possible to directly relate optical information to the structural properties.

up by the near-field probe at each position on the sample surface is interferometrically mixed with light split from the same laser source that has propagated along the reference branch of the setup (Fig. 1). Heterodyne detection of the interference signal, established by acousto-optical modulation in the reference branch, then enables a separation of amplitude and phase information by a dual-output lock-in amplifier (LIA) [27]. As the phase and time information of the evanescent field, at each position on the sample, is the same as that of the propagating wave we obtain direct information on the propagating light field.

To visualize dynamic effects, we launch femtosecond pulses $(\lambda=1300 \pm 2 \mathrm{~nm})$, generated by a Ti:sapphire-pumped optical parametric oscillator (Spectra-Physics Opal), into our model system. The arrival time (12.5 ns) between subsequent laser pulses is long enough so that the pulse picked up by the probe can only interfere with part of the same laser pulse that has propagated along the reference branch. The pulse duration of the input field $\widetilde{E}(t)$ is measured by a conventional background free intensity autocorrelation technique and yields a full width at half maximum (FWHM) of the pulse amplitude of $123 \pm 3$ fs [3]. Note that when using ultrashort pulses optical interference will only occur when temporal overlap exists between the pulse in the signal and 
the pulse in the reference branch at the point where the branches are brought together. An optical delay line in the reference branch completes the pulse tracking setup. Basically the position of the optical delay line determines the length of the reference branch and thus defines a reference time for the measurement. More details regarding the experimental setup ${ }^{1}$ can be found elsewhere $[27,30]$.

As a model system for our measurements we have used a $\mathrm{Si}_{3} \mathrm{~N}_{4}$ planar channel waveguide fabricated in a $\mathrm{Si}_{3} \mathrm{~N}_{4} / \mathrm{SiO}_{2}$ layer system on a $\mathrm{Si}$ substrate. The experimentally determined slab thickness, width, and height of the structure are $170 \pm 5 \mathrm{~nm}, 3.1 \pm 0.1 \mu \mathrm{m}$, and $39 \pm 3 \mathrm{~nm}$, respectively. Linearly polarized light has been coupled into the channel waveguide with a polarization parallel to the sample plane, such that the $\mathrm{TE}_{00}$ and $\mathrm{TE}_{01}$ mode are excited. These are the only two TE polarized modes supported by the waveguide for the measured waveguide parameters, as determined by an effective index method. The TM polarized mode, which is in principle supported by the waveguide, is not excited. All measurements are performed at a distance of $12 \pm 0.5 \mathrm{~mm}$ away from the incoupling facet. All data collection has been performed by raster scanning an uncoated fiber probe while keeping the probe-sample distance constant with the fast axis along the waveguide channel (line frequency $=0.098 \mathrm{~Hz}$ ), with a fixed position of the optical delay line in the reference branch for each image.

\section{PULSE TRACKING IN A BIMODAL WAVEGUIDE}

A local time resolved heterodyne interference measurement on a bimodal waveguide is presented in Fig. 2 for a fixed position of the optical delay line. Figure 2(a) shows the topography, which is collected simultaneously with the optical information. The detected LIA signal is proportional to the measured optical amplitude $(\mathcal{A})$ times the cosine of the phase of the optical interference signal $(\cos \Phi)$. From this measurement the optical amplitude and the cosine of the phase of the optical signal can be separated, as presented in Figs. 2(b) and 2(c), respectively [27].

The amplitude in Fig. 2(b) shows a roughly Gaussian shape with a clear spatial beating pattern at the pulse front. The appearance of this beating is easily explained by the fact that the $\mathrm{TE}_{00}$ and $\mathrm{TE}_{01}$ modes have different propagation constants leading to a spatial mode beat. A Fourier transform of the $\mathcal{A} \cos \Phi$ signal along the waveguide, as given in Fig. 3 (a), reveals a periodicity of the fringes of $858 \pm 3 \mathrm{~nm}$ and $875 \pm 5 \mathrm{~nm}$ corresponding to the $\mathrm{TE}_{00}$ and $\mathrm{TE}_{01}$ modes, respectively. These measured wavelengths inside the structure immediately yield the phase velocity of the light in the waveguide and thus the effective indices of refraction. In this way we obtain effective indices of $1.52 \pm 0.02$ and $1.49 \pm 0.03$ for the $\mathrm{TE}_{00}$ and $\mathrm{TE}_{01}$ modes, respectively. These values are in

\footnotetext{
${ }^{1}$ In the current experiment the optical powers in the signal and reference branch are approximately $1 \mathrm{nW}$ and $1 \mu \mathrm{W}$. As a result we operate several orders of magnitude above the detection limit of the instrument. More details regarding experimental sensitivity and related issues are beyond the scope of this paper and will be published elsewhere.
}

reasonable agreement with the calculated values of 1.56 and 1.53. The slight difference in accuracy between the experimental values results from the large difference in the amplitudes of the $\mathrm{TE}_{00}$ and $\mathrm{TE}_{01}$ modes.

In the optical amplitude in Fig. 2(b) it is visible that the beating pattern occurs only towards the right side of the image. An inverse Fourier transform of the relevant peaks of the Fourier spectra for the individual modes yields the amplitude for the $\mathrm{TE}_{00}$ and $\mathrm{TE}_{01}$ modes as given in Figs. 2(d) and 2(e), respectively. We observe that the $\mathrm{TE}_{01}$ mode is in front of the $\mathrm{TE}_{00}$ mode. This separation results from the fact that different modes in a photonic structure have different group velocities. The origin of the fact that the $\mathrm{TE}_{01}$ is found to have a larger group velocity can be found in the fact that this mode is further extended into the air and therefore has a larger group velocity.

From the Fourier filtered amplitudes presented in Figs. 2(d) and 2(e) we can directly retrieve the mode profiles, as depicted in Fig. 3(b). Figure 3(c) shows the simultaneously obtained topography information along such a cross section. The arrows in Fig. 3(b) indicate the FWHM for the individual mode profiles, which are $2.08 \pm 0.07 \mu \mathrm{m}$ and $4.00 \pm 0.12 \mu \mathrm{m}$ for the $\mathrm{TE}_{00}$ and $\mathrm{TE}_{01}$ modes, respectively. The maximum amplitude of the $\mathrm{TE}_{00}$ mode is $11.8 \pm 0.5$ times higher than the amplitude of the $\mathrm{TE}_{01}$ mode. Furthermore, a line trace along the waveguide for the individual modes shows the shape of the pulses, as depicted in Fig. 3(d). Fitting a Gaussian distribution, as shown by the black and red line, yields a pulse length as $58.9 \pm 0.6 \mu \mathrm{m}$ and $70.6 \pm 7.1 \mu \mathrm{m}$ for the $\mathrm{TE}_{00}$ and $\mathrm{TE}_{01}$ modes, respectively. Obtaining an accurate fit for the $\mathrm{TE}_{01}$ mode for this specific measurement is difficult due to the relatively low optical amplitude of this signal that results in a relatively low signal to noise ratio. Nevertheless, the presented Fourier filtered amplitude data clearly demonstrate that all relevant parameters for the optical amplitude of an individual mode can still be retrieved.

In Figs. 4(b)-4(j) the measured optical amplitudes on the waveguide depicted in Fig. 4(a) are shown for nine subsequent measurements with increasing reference times. Between each measurement, the reference time is shifted $200 \pm 2 \mathrm{fs}$ by lengthening the reference branch by $60.0 \pm 0.6 \mu \mathrm{m}$. For each reference time a similar pattern as in Fig. 2(b) is found, but further along the waveguide. Note that the beating between the two modes prevents a pinpointing of the position of the individual pulses directly in these images $[29,30]$. However, by applying a Fourier filter to the raw data for the entire time sequence, we can separate the contribution of the $\mathrm{TE}_{00}$ mode in time as depicted in Fig. 5. The linear dependence of the position of the pulse in the waveguide as a function of the reference time yields a group velocity of $1.46 \pm 0.04 \times 10^{8} \mathrm{~m} / \mathrm{s}$.

A similar approach for the $\mathrm{TE}_{01}$ yields a lower group velocity than for the $\mathrm{TE}_{00}$ mode, which is obviously not correct. It turns out that the reduced spatial resolution used to obtain the time sequence results in a reduced spectral separation of the modes. As a result a large part of the $\mathrm{TE}_{00}$ mode is mixed in the $\mathrm{TE}_{01}$ amplitude, which makes the determination of the group velocity for the $\mathrm{TE}_{01}$ mode inaccurate. However, we know that upon propagation of $12 \pm 0.5 \mathrm{~mm}$ 

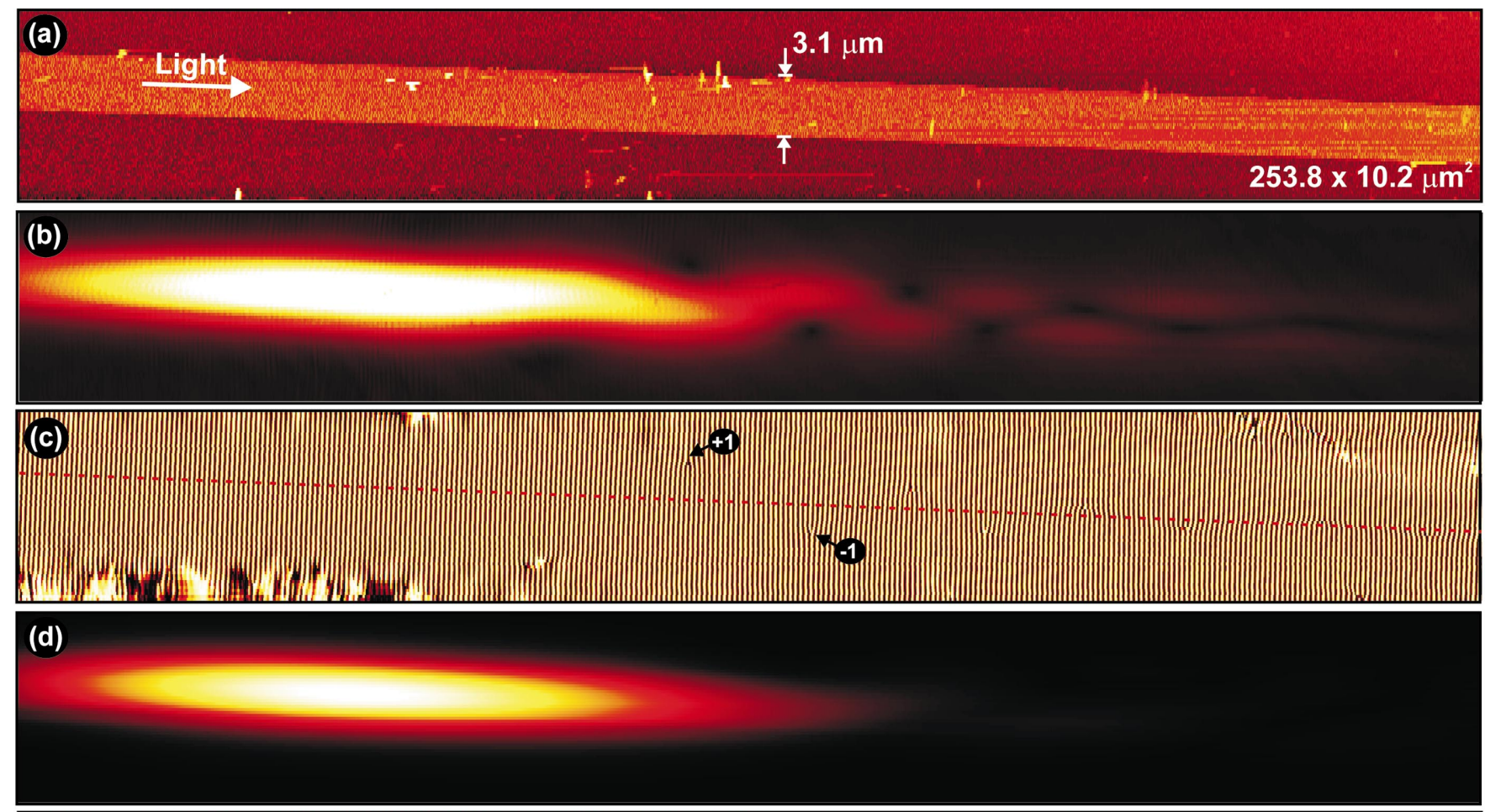

(e)

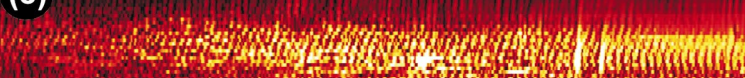

Wfor

FIG. 2. (Color) A time resolved heterodyne interference PSTM measurement on a bimodal $\mathrm{Si}_{3} \mathrm{~N}_{4}$ channel waveguide for a fixed position of the optical delay (image size: $253.8 \times 10.2 \mu \mathrm{m}^{2}$ ). Linear polarized light has been coupled in the waveguide such that only the $\mathrm{TE}_{00}$ and $\mathrm{TE}_{01}$ modes are excited. (a) The topography of the waveguide. The measured height and width of the waveguide are $39 \pm 3 \mathrm{~nm}$, and $3.1 \pm 0.1 \mu \mathrm{m}$. The slab thickness is determined to be $170 \pm 5 \mathrm{~nm}$. (b) The optical amplitude of the pulse while propagating through the waveguide as derived from the measured LIA signal as a function of the lateral position in the plane of the sample. It is apparent that the amplitude is confined to the waveguide. A clear beating pattern due to multiple propagating modes is observed at the right side of the image. (c) The corresponding $\cos \Phi$ of the optical interference. The red dotted line depicts the center of the waveguide. As a result of the beating between different modes phase singularities occur at the position of zero amplitude. These singularities consist of pairs with opposite topological charge (indicated by arrows). It is striking to observe that the distance between the singularities is not constant, in sharp contrast to measurements performed with continuous wavelength laser sources. (d) The optical amplitude of the TE 00 as retrieved from the LIA data through Fourier filtering. (e) Optical amplitude of the $\mathrm{TE}_{01}$ mode. We observe that the $\mathrm{TE}_{01}$ is clearly in front of the $\mathrm{TE}_{00}$ mode, which demonstrates that the $\mathrm{TE}_{01}$ mode travels faster in the waveguide structure.

through the waveguide the modes are separated only by $83 \pm 5 \mu \mathrm{m}$. As a result we have a direct measure for the $\Delta v_{g}$ between the two modes. Therefore the average group velocity for the $\mathrm{TE}_{01}$ mode is estimated to be $1.47 \pm 0.04$ $\times 10^{8} \mathrm{~m} / \mathrm{s}$, which is $0.7 \%$ larger than the measured speed of the $\mathrm{TE}_{00}$ mode. Note, however, that the value for the $\mathrm{TE}_{01}$ mode is an averaged value for the whole waveguide from incoupling to the pulse position and not a locally determined velocity.

The observed beating pattern as is visible in Fig. 2(b) leads to several interesting effects. For example, a closer look at the optical phase presented in Fig. 2(c) reveals phase singularities at positions where the field amplitude vanishes in Fig. 2(b), as indicated by the arrows in Fig. 2(c). The vanishing optical amplitude at these positions is a direct result of the interference between the $\mathrm{TE}_{00}$ and $\mathrm{TE}_{01}$ modes in the waveguide. At positions with equal amplitude, but opposite phase, these two modes give rise to destructive interference explaining the appearance of the singularities [25]. Singularities with a topological charge of +1 are observed on one side of the red dotted line in Fig. 2(c), while at the other side a charge of -1 is found. Note that the assignment of the sign of the topological charge for this geometry is arbitrary, but it needs to be opposite for singularities that are each other's mirror image. The two interfering modes both consist of a two-dimensional Gaussian-Hermitian distribution as is visible in Figs. 3(b) and 3(d) for the directions perpendicular to and along the waveguide, respectively. Be aware that the absolute amplitude for the $\mathrm{TE}_{01}$ is depicted in Fig. 3(b), so that the normal peak-dip shape of the first order mode shows up as two peaks. By using these two-dimensional distributions it is easily understood why the singularities move to- 


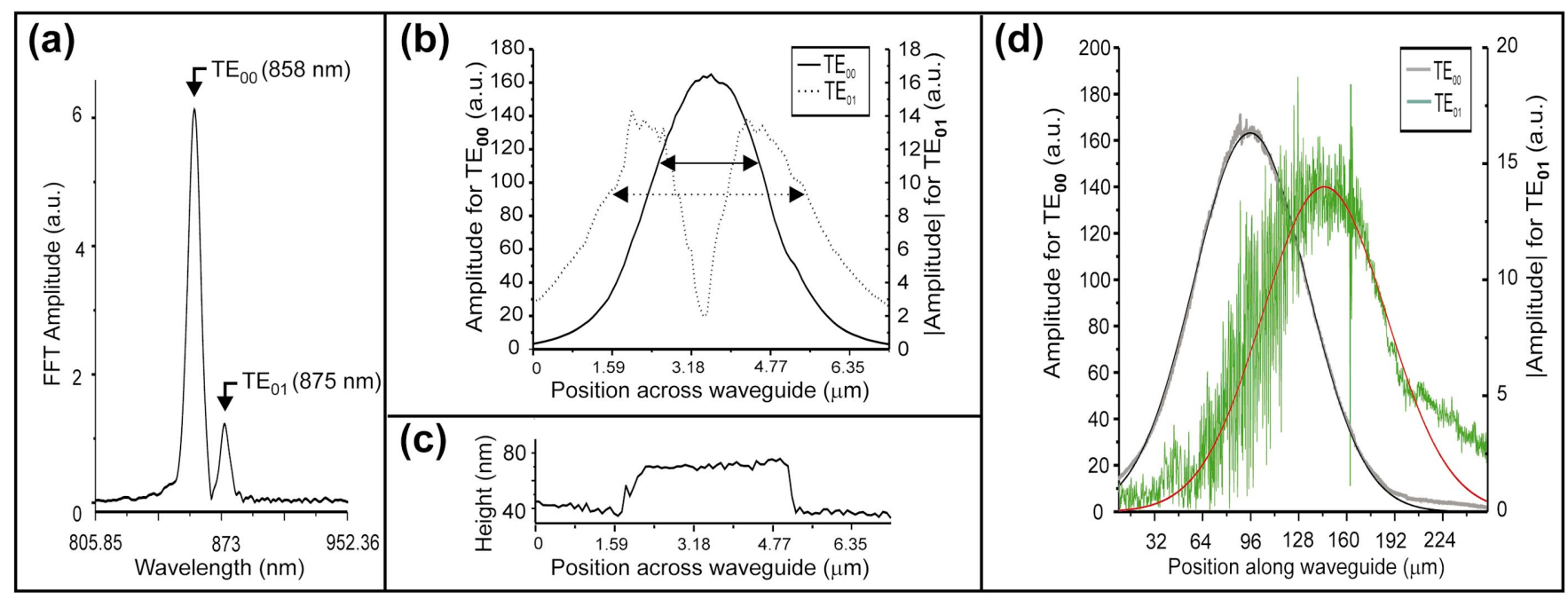

FIG. 3. (Color) (a) A Fourier transform of the $\mathcal{A} \cos \Phi$ signal along the waveguide. The positions of the maxima immediately yield the phase velocity for the respective modes and hence the effective indices of $1.52 \pm 0.02$ and $1.49 \pm 0.03$ for the $\mathrm{TE}_{00}$ and $\mathrm{TE}_{01} \mathrm{modes}$ respectively. Note that the $\mathrm{TE}_{01}$ is very weak compared to the $\mathrm{TE}_{00}$ mode. (b) The mode profile for each individual mode measured by taking a cross section through the waveguide at the maximum amplitude of the individual modes in Figs. 2(d) and 2(e), respectively. (c) The simultaneously acquired topography along such a cross section. (d) A line trace along the waveguide which shows the pulse shape for the respective modes. The FWHM of the pulse profile is determined by the GVD in both the signal and reference branch. Fitting a Gaussian line shape (black and red line) for the respective modes therefore allows a retrieval of the difference in GVD between the two modes.

wards the center of the waveguide as indicated by the red dotted line in Fig. 2(c). This is schematically illustrated in Fig. 6(a) by depicting the amplitude of both a $\mathrm{TE}_{00}$ and $\mathrm{TE}_{01}$ mode in an isoline representation. Positions where the amplitude of both modes is equal are indicated by the red lines which clearly move towards the center of the waveguide. The first requirement for singularities is that they only occur at location of equal amplitude. As a result the singularities clearly move towards the center of the waveguide.

The other requirement for a singularity is that the interfering waves have opposite phase. This is schematically illustrated for a Fourier limited pulse, which has a single carrier wavelength in Fig. 6(b). Depicted are lines where the phase for the modes is 0 (solid lines) and $\pi$ (dotted lines). Green is used for the $\mathrm{TE}_{00}$ mode, while blue represents the $\mathrm{TE}_{01}$ mode which has a longer wavelength. Note that at the center of the waveguide, depicted by the black line, a phase jump of $\pi$ occurs for the $\mathrm{TE}_{01}$. At regular intervals the dotted and solid lines for the respective modes coincide, as indicated by the depicted arrows, representing lines of opposite phase. By depicting schematically the red line from Fig. 6(a) for locations of equal amplitude in this figure, we see that the crosses define a location where both requirements for the occurrence of a phase singularity are met. It is important to realize that in discussing the phase singularities the amplitude and phase information can be considered separately. Along a line of opposite phase we just have to look for a location where the amplitudes are equal.

Based on the explanation given in Fig. 6 the phase singularities might be expected at equidistant intervals determined by the difference in effective index for the two modes. It is therefore striking to observe that the distance between individual singularities is not constant in the measured data as can be seen in Fig. 2(c). This is shown in more detail in Fig.
7, where the distance between subsequent phase singularities is plotted as a function of the reference time. In this graph, the letter assignment corresponds to the images presented in Fig. 4. On the horizontal axis the number of a singularity is plotted. In this the first singularity visible in Fig. 4(b), as indicated by the arrow, is used as the reference point and numbered as 1 . Note that for subsequent singularities the phase difference between the two modes increases or decreases by $\pi$. Based on the measured center wavelengths of $858 \pm 3 \mathrm{~nm}$ and $875 \pm 5 \mathrm{~nm}$ for the $\mathrm{TE}_{00}$ and $\mathrm{TE}_{01}$ a phase singularity separation of $22 \mu \mathrm{m}$ is therefore expected. Clearly, separations that are both longer and shorter than $22 \mu \mathrm{m}$ are observed in the measurement. From Fig. 7 it is clear that the distance between individual singularities not only changes within one measurement frame, but also depends strongly on the reference time. These effects are in sharp contrast with results obtained for continuous wavelength sources where these distances are only determined by the effective wavelengths of the modes and therefore constant [25]. The difference is directly related to the observation of the propagation of ultrashort pulses with a resulting finite bandwidth. Pulses experience group velocity dispersion (GVD) upon propagation through a dispersive medium which is the origin of this observation.

\section{EFFECT OF DISPERSION}

To understand the observed beating patterns we have to account for pulses with a finite bandwidth. In the case of a Fourier limited pulse only the central wavelength would contribute to the interference. As a result regularly spaced singularities would be observed which is clearly not the case. We need to take into account the fact that the actual pulses generating the interference signal are no longer Fourier lim- 


\section{(a)}

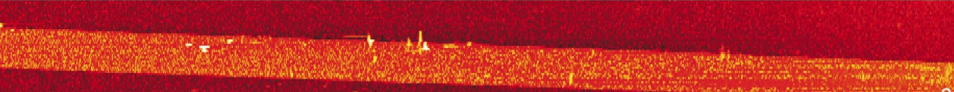
$253.8 \times 10.2 \mu \mathrm{m}^{2}$
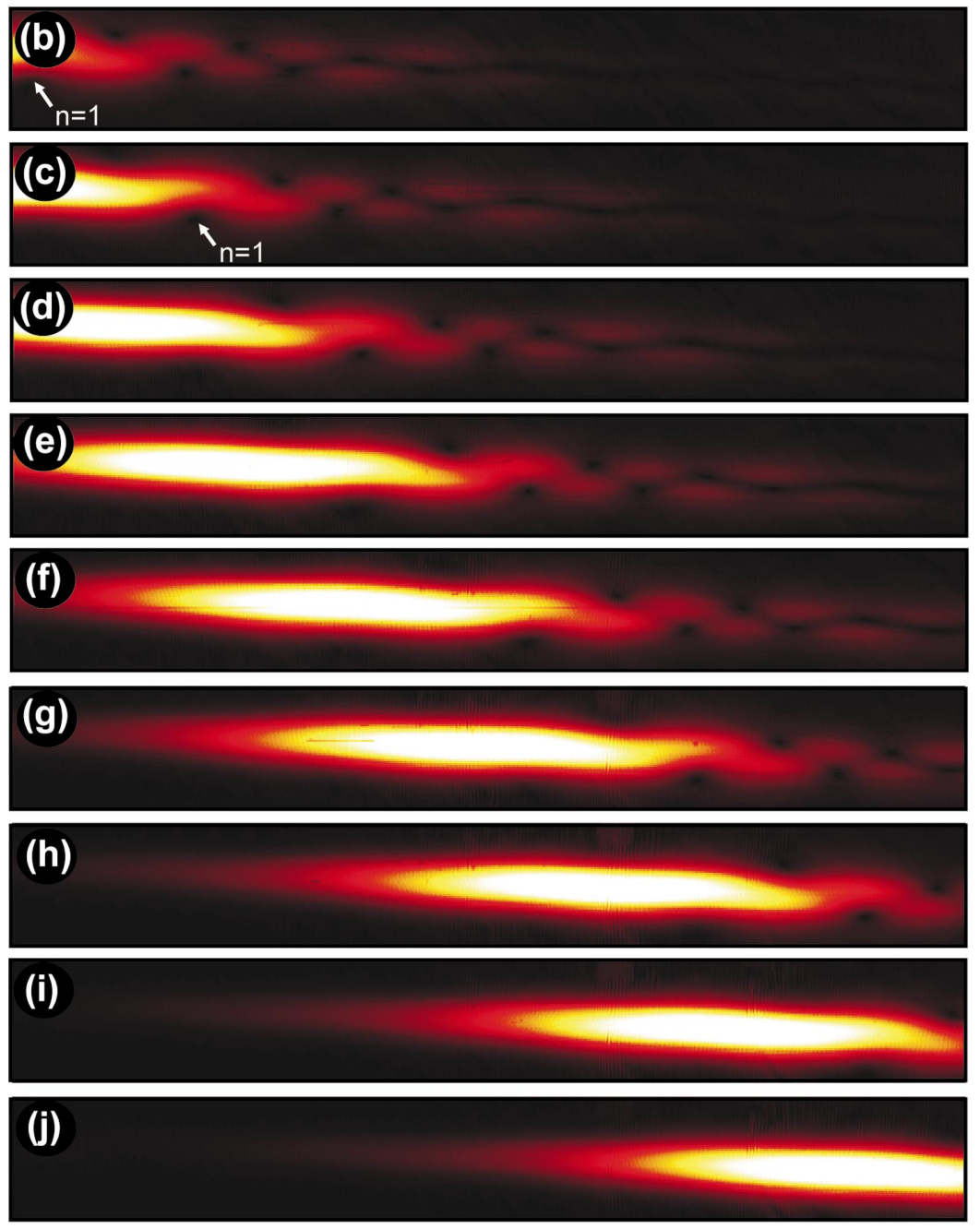

FIG. 4. (Color) Pulse tracking experiment for different reference times. Linear polarized light has been coupled in the waveguide to excite the $\mathrm{TE}_{00}$ and $\mathrm{TE}_{01}$ modes. (a) The simultaneously measured topography (image size: $\left.253.8 \times 10.2 \mu \mathrm{m}^{2}\right)$. [(b)-(j)] The optical field amplitude as measured by the instrument for nine different positions of the optical delay line. From (b)-(j) the optical path length of the reference branch is increased in steps of $60 \pm 0.6 \mu \mathrm{m}$. This results in steps of the reference time of $200 \pm 2$ fs. For each frame a similar pattern as in Fig. 2(b) is found, but further along the waveguide. Note that the occurrence of a beating between the modes prevents direct pinpointing of the position of the individual pulses in these images.

ited due to changes in shape resulting from propagation through a dispersive medium. Consequently, it might be expected that the measurement of the phase singularities yields direct information on the local dispersion characteristics. In the following, we will show how this allows the retrieval of the GVD for the individual modes by means of a refinement of our analytical model of the time-resolved PSTM measurements [30].

The standard way in which dispersion is measured is via interferometry [3]. In interferometry, the "dispersive" system of which the dispersion is to be measured is placed in one arm of the interferometer exactly as in the time resolved experiments presented in this paper. By dispersive in this context we mean any linear system in which the propagation constant $\beta(\omega)$ is a function of frequency. It is assumed that the dispersive effects are adequately described in terms of phase and group velocity and the group velocity dispersion. Therefore $\beta(\omega)$ is written as a second order Taylor expansion about its value at $\omega_{0}$ with the derivatives $\beta^{\prime} \equiv d \beta / d \omega$ and $\beta^{\prime \prime} \equiv d^{2} \beta / d \omega^{2}$ evaluated at $\omega=\omega_{0}$.

This $\beta(\omega)$, which defines the dispersion characteristics for a mode, is exactly the property we would like to measure locally. Quite naturally we can write $v_{g}\left(\omega_{0}\right) \equiv 1 / \beta^{\prime}\left(\omega_{0}\right)$ and $v_{\phi} \equiv \omega_{0} / \beta\left(\omega_{0}\right)$ for the locally measured group and phase velocity, respectively. We recently published an analytical model to interpret the results obtained with a time-resolved PSTM in the case of single mode propagation [30]. By extending this model we will show how the different GVD of the individual modes can be retrieved from the observed beating pattern.

A Gaussian light pulse after traveling an arbitrary distance $z$ through a dispersive medium can be written as $[3,30]$

$$
\widetilde{E}(z, t)=\sqrt{\frac{\tilde{\Gamma}(z)}{\tilde{\Gamma}_{0}}} \exp \left[i \omega_{0}\left(t-\frac{z}{v_{\phi}}\right)-\tilde{\Gamma}(z)\left(t-\frac{z}{v_{g}}\right)^{2}\right],
$$

where the pulse propagation parameter $\widetilde{\Gamma}=a-i b$ is related to the pulse width through the parameter $a$, while the parameter $b$ is a measure for the frequency chirp on the pulse. The input 

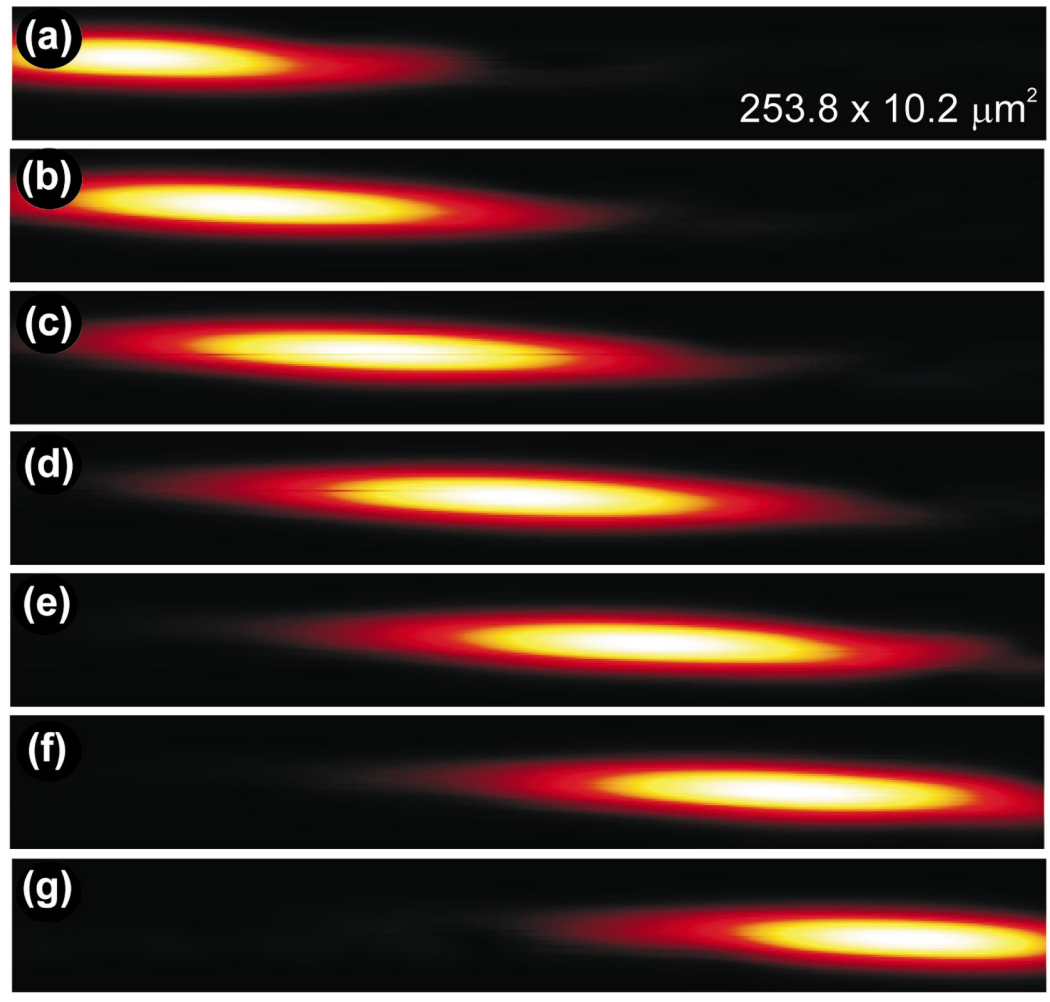

FIG. 5. (Color) The optical amplitude for the $\mathrm{TE}_{00}$ mode as derived by applying a Fourier filter on the raw data of Figs. 4(d)-4(j). This result shows that the position of the $\mathrm{TE}_{00}$ pulse at a reference time can be pinpointed in space. The pulses can be seen to propagate through the structure as a function of time giving a direct local measurement of the group velocity for this mode. By determining the "center of mass" for each frame a local value of the group velocity $v_{g}$ for the pulse of $1.46 \pm 0.04$ $\times 10^{8} \mathrm{~m} / \mathrm{s}$ is found. pulse parameter $\widetilde{\Gamma}_{0}$ is related to the initial pulse duration $\tau_{p}$ $=[2 \ln (2) / a]^{1 / 2}$, while $b=0$ as we use unchirped Fourier limited input pulses $\left(\widetilde{\Gamma}_{0} \in \mathbb{R}\right)$. Upon propagation through a dispersive medium of length $z$ the output pulse is still a Gaussian pulse, but the pulse propagation parameter $\Gamma(z)$ is changed as $1 / \widetilde{\Gamma}(z)=1 / \widetilde{\Gamma}_{0}+2 i \beta^{\prime \prime} z$ due to the GVD. From Eq. (1) it is clear that the carrier frequency within the pulse propagates at the phase velocity $v_{\phi}$, while the pulse envelope itself propagates at the group velocity $v_{g}$ evaluated at the center of the pulse spectrum. The pulse envelope changes in shape with distance and develops a chirp due to the GVD.

The time-resolved PSTM is considered as a MachZehnder type interferometer with heterodyne detection as schematically shown in Fig. 8. The input field $\widetilde{E}(t)$, assuming an unchirped input pulse $\left(\widetilde{\Gamma}_{0} \in \mathbb{R}\right)$, is split by the beam splitter into fields that travel through the two arms of the interferometer. The field through the sample is designated $\widetilde{E}_{s}(z, t)$, and the delayed field through the reference branch is called $\widetilde{E}_{r}(t-\tau)$. The $z$ dependence is explicitly included to represent the fact that the near-field probe is moving along the sample and thus includes additional dispersive medium as a function of position, while $\tau$ represents the optical delay through air in the reference branch in the usual fashion. Note that the inclusion of additional dispersive medium as a function of the probe movement is the crucial difference with conventional phase sensitive time-resolved interferometry where the delay line moves in vacuum $[3,33,34]$.

By using Eq. (1) for a pulse propagating through the dispersive media in the respective branches of the interferometer, we obtained for the measured LIA signal [30]

$$
\begin{aligned}
V_{1}\left(z_{s}, \tau\right) \propto & A \int_{-\infty}^{\infty} \exp \left[-\Gamma_{r}\left(t-\xi_{r}\right)^{2}-\Gamma_{s}\left(t-\xi_{s}\right)^{2}\right] \\
& \times \cos \left\{\Upsilon+\kappa_{r} \Gamma_{r}\left(t-\xi_{r}\right)^{2}+\kappa_{s} \Gamma_{s}\left(t-\xi_{s}\right)^{2}\right\} d t
\end{aligned}
$$

where

$$
\begin{gathered}
\kappa_{r}=2 \beta_{r}^{\prime \prime} z_{r} \Gamma_{0} ; \quad \kappa_{s}=-2 \beta_{s}^{\prime \prime} z_{s} \Gamma_{0}, \\
\Gamma_{r}=\frac{\Gamma_{0}}{1+\left(\kappa_{r}\right)^{2}} ; \quad \Gamma_{s}=\frac{\Gamma_{0}}{1+\left(\kappa_{s}\right)^{2}}, \\
A=\frac{A_{r} A_{s}}{\sqrt{\Gamma_{0}}} \sqrt[4]{\Gamma_{r} \Gamma_{s}}, \\
\xi_{r}=\tau+\frac{z_{r}}{v_{g r}} ; \quad \xi_{s}=\frac{z_{s}}{v_{g s}},
\end{gathered}
$$

and

$$
\Upsilon=-\frac{\arctan \left(\kappa_{r}\right)+\arctan \left(\kappa_{s}\right)}{2}-\omega_{0}\left(\tau+\frac{z_{r}}{v_{\phi r}}-\frac{z_{s}}{v_{\phi s}}\right) .
$$

Here, the $A$ corresponds to an amplitude with $A_{i}$ constants that are proportional to the different optical power density in the two branches, $\Gamma_{i}$ corresponds to the real part of a modified pulse propagation parameter, $\xi_{i}$ represents an optical path length, $\kappa_{i}$ is a GVD dependent parameter, and $Y$ is simply a time-independent variable in the optical phase. The subscript $i$ denotes the reference and signal branches, respec- 


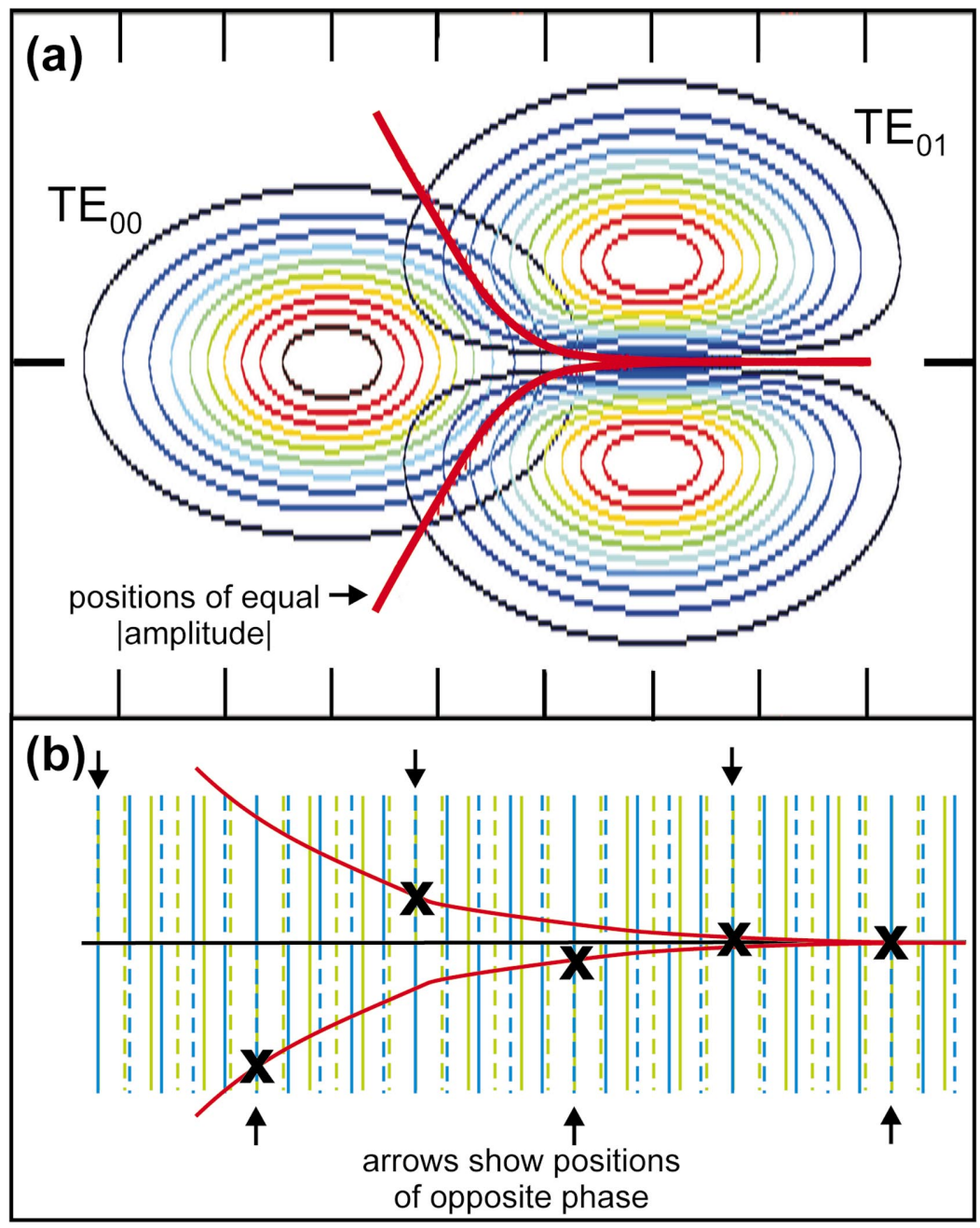

FIG. 6. (Color) (a) Schematic drawing of the amplitude of both a $\mathrm{TE}_{00}$ and a $\mathrm{TE}_{01}$ mode in an isoline representation. Positions where the amplitude for both modes is equal are indicated by the red lines. Singularities can only occur at locations where the amplitude is equal. Therefore it is clear that singularities move to the center of the waveguide. (b) For a Fourier limited pulse the two interfering modes consist of two discrete periods. Green is used for the $\mathrm{TE}_{00}$ mode, while blue represents the $\mathrm{TE}_{01}$ mode which has a longer wavelength. Depicted are lines where the phase for the modes is 0 (solid lines) and $\pi$ (dotted lines). Note that at the center of the waveguide, depicted by the black line, a phase jump of $\pi$ occurs for the $\mathrm{TE}_{01}$ mode. At regular intervals the dotted and solid lines for the respective modes coincide, as indicated by the depicted arrows, representing lines of opposite phase. Depicting the red line for locations of equal absolute amplitude shows that a phase singularity will occur at the locations indicated by the crosses. Note that the phase singularities occur at equidistant intervals when the phase difference $\Delta \Phi$ between the modes is equal to $\pi$.

tively. The $z_{i}$ represents the total length of dispersive medium in either branch.

In our case different dispersive media are present in the respective branches of the interferometer. Although not written down explicitly, this is included in the model by multiplying the original spectrum with the different frequency dependent propagation constants for the respective media. The result is simply a summation over the different propagation constants. By solving integral equation (2a), we obtain the following expressions for the amplitude and phase of the interference signal as it is measured by the time-resolved PSTM [30]:

$$
\mathcal{A}\left(z_{s}, \tau\right)=\frac{A_{r} A_{s} \sqrt{\pi / \Gamma_{0}}}{\sqrt[4]{4+\left(\kappa_{r}+\kappa_{s}\right)^{2}}} \exp \left[\frac{-2 \Gamma_{0}\left(\xi_{r}-\xi_{s}\right)^{2}}{4+\left(\kappa_{r}+\kappa_{s}\right)^{2}}\right],
$$

and

$$
\begin{aligned}
\Phi\left(z_{s}, \tau\right)= & \Upsilon+\frac{1}{2} \arctan \left\{\frac{\Gamma_{r} \kappa_{r}+\Gamma_{s} \kappa_{s}}{\Gamma_{r}+\Gamma_{s}}\right\} \\
& -\left\{\frac{\Gamma_{0}\left(\xi_{r}-\xi_{s}\right)^{2}\left[\kappa_{r}+\kappa_{s}\right]}{4+\left(\kappa_{r}+\kappa_{s}\right)^{2}}\right\},
\end{aligned}
$$

where the variables with the subscript $s$ are the ones that change as a function of the position of the probe. In the experiment only $z_{s}$ and $\tau$ are varied. However, the optical delay line is kept at a fixed position for each individual measurement, so that $\tau$ is in fact constant (both $\xi_{r}$ and $Y$ depend on $\tau$ ).

With Eqs. (3) and (4), we have analytical expressions for both the measured pulse phase and amplitude as a function of position for the case of single mode behavior. For the case of multimode devices the present model has to be extended to incorporate multimode propagation to fully understand the results measured with the time-resolved PSTM. This is done by replacing the electromagnetic field in the sample branch $\widetilde{E}_{s}(t)$ by a summation over independent orthogonal modes. It is then straightforward to show that the integral in Eq. (2a) is transformed into

$$
\begin{aligned}
V_{1}\left(z_{s}, \tau\right) \propto & A_{r} \int_{-\infty}^{\infty} \sum_{n=0}^{n=N} \frac{A_{s n}}{\sqrt{\Gamma_{0}}} \sqrt[4]{\Gamma_{r} \Gamma_{s n}} \exp \left[-\Gamma_{r}\left(t-\xi_{r}\right)^{2}\right. \\
& \left.-\Gamma_{s n}\left(t-\xi_{s n}\right)^{2}\right] \cos \left\{\Upsilon_{n}+\kappa_{r} \Gamma_{r}\left(t-\xi_{r}\right)^{2}\right. \\
& \left.+\kappa_{s n} \Gamma_{s n}\left(t-\xi_{s n}\right)^{2}\right\} d t
\end{aligned}
$$

Here, the $n$ represents the mode number while $N$ defines the total number of modes present. The variables with the subscript $s n$ are defined corresponding to the definitions in Eq. 


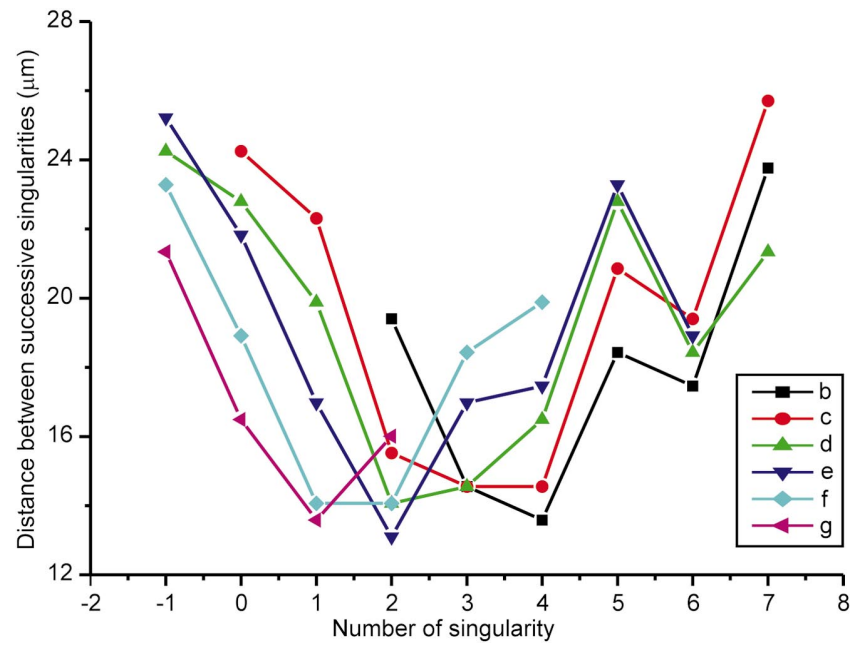

FIG. 7. (Color) Measured distance between subsequent phase singularities as a function of reference time. The horizontal axis depicts the number of a singularity, which corresponds to an effective phase difference between the two modes of $\pi$ as illustrated in Fig. 6(b). The first singularity visible in Fig. 4(b) (see arrow) is used as the reference point and numbered as 1 . Subsequent measurements are lettered corresponding to the frames used in Fig. 4. It is striking to observe that the distance between individual singularities varies within one measurement frame in sharp contrast with the expectation put forward in Fig. 6. Furthermore, this separation between singularities also shows a dependence on the reference time.

(2b) for each individual mode separately. The single integral in Eq. (2a) is replaced by an integration over a sum of orthogonal modes. As integrating is a linear operation the sequence of integrating and summation can be interchanged for the typically continuous functions representing electrical fields. Therefore we can solve the integral for each individual mode and subsequently perform a coherent summation over

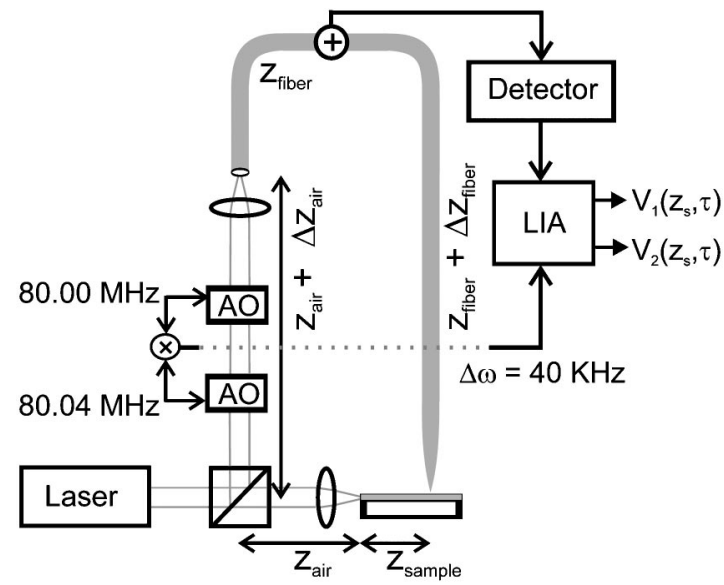

FIG. 8. Mach-Zehnder model for the time resolved photon scanning tunneling microscope. The fiber length difference $\Delta z_{\text {fiber }}$ between the two arms is compensated by the air path length difference $\Delta z_{\text {air }}$. Heterodyne detection is applied, using acousto-optic modulation of the light in the reference branch. The resulting interference signal is measured with a LIA and allows one to extract the amplitude and phase of the local optical field: AO, acousto-optic modulator; and LIA, lock-in amplifier. all solutions. In other words: we can use the equations for the amplitude and optical phase as given in Eqs. (3) and (4) for each individual mode. A coherent summation over the solutions for all modes present in the photonic structure using these equations will subsequently give the solution for the resulting optical amplitude and phase as observed by the time-resolved PSTM.

\section{RETRIEVAL OF THE GVD FOR INDIVIDUAL MODES IN BIMODAL STRUCTURES}

In this section we will use the presented analytical model to show how the local GVD for individual modes can be retrieved from the experimental data. In the experiment the photon-tunneling signal and the reference signal recombine in a 50/50 fiber coupler after propagating through different lengths of single mode fibers and other bulk optical glass components. The pulse in the signal branch travels $12 \pm 0.5 \mathrm{~mm}$ through the sample and $68.5 \pm 0.5 \mathrm{~cm}$ through dispersive fibers and other glass components, and $23.5 \pm 0.2 \mathrm{~cm}$ through air. For the reference branch these distances are $38.5 \pm 0.5 \mathrm{~cm}$ and $75.6 \pm 0.5 \mathrm{~cm}$ for the air and dispersive materials, respectively. Note that in the reference branch the path length in air can be changed as a function of the position of the optical delay line [delay $\tau$, Eq. (2)], while in the sample arm the length of the (dispersive) sample $z_{s}$ changes as a function of the probe position.

In order to detect the interference signal the optical path lengths in the respective branches have to be made equal. Therefore the fiber length difference $\Delta z_{\text {fiber }}$ between the branches is balanced by adding an extra air path $\Delta z_{\text {air }}$ in the reference branch of the interferometer as depicted in Fig. 8. This air path is also dispersive, but the dispersion of air is so small compared to the dispersion of fibers at these wavelengths that it can be neglected. If we consider the interferometer as a linear system, we can simply replace the different dispersive elements, excluding the sample(!), in either branch by a single effective dispersive element, indicated as a fiber. As a result the system can be modeled using single dispersive elements in both branches together with a dispersive sample in the signal branch as schematically depicted in Fig. 8. With this the amplitude and phase maps can be calculated using the experimentally obtained parameters. Although Eqs. (3) and (4) describe propagation in one dimension, two dimensional maps can be calculated by using the modal distribution as depicted in Fig. 3(b) for each horizontal line as input. This approach is valid for systems where the different modes are sufficiently far separated in Fourier space to allow retrieval of the individual mode profiles.

Time-resolved PSTM allows a direct determination of the central wavelength and the group velocity for individual modes. As a result, the $\beta$ and $\beta^{\prime}$ are also known for both modes. However, it may be expected that the pattern of phase singularities and its time dependence as presented in Fig. 7 yield direct information on the GVD. The positions at which singularities occur are a direct measure for the phase difference between the modes. Be aware that the exact experimental configuration influences the observed optical phase and as such determines which of the terms in Eq. (4) 
are dominant. Insertion of our experimental parameters into Eq. (4) shows that in our experiment the observed phase is dominated by the last term of Eq. (2c) and the last term of Eq. (4), respectively. As a result we can write

$$
\Phi\left(z_{s}, \tau\right) \cong-\omega_{0}\left(\tau+\frac{z_{r}}{v_{\phi r}}-\frac{z_{s}}{v_{\phi s}}\right)-\left\{\frac{\Gamma_{0}\left(\xi_{r}-\xi_{s}\right)^{2}\left[\kappa_{r}+\kappa_{s}\right]}{4+\left(\kappa_{r}+\kappa_{s}\right)^{2}}\right\},
$$

for the observed optical phase from the individual modes. Note the similarity between the second term in this equation and the exponent in Eq. (3). Clearly, this second term can be fully defined with the parameters recovered from the measured optical amplitude for the individual modes. As a result, the measured phase fringes give complete information about the dispersion relation as can be seen when writing Eq. (6) in terms of $\beta$. This yields

$$
\begin{aligned}
\Phi\left(z_{s}, \tau\right) \cong & -\omega_{0} \tau-\beta_{r} z_{r}+\beta_{s} z_{s} \\
& -\left\{\frac{2 \Gamma_{0}^{2}\left(\tau+\beta_{r}^{\prime} z_{r}-\beta_{s}^{\prime} z_{s}\right)^{2}\left[\beta_{r}^{\prime \prime} z_{r}-\beta_{s}^{\prime \prime} z_{s}\right]}{4+\left[2 \Gamma_{0}\left(\beta_{r}^{\prime \prime} z_{r}-\beta_{s}^{\prime \prime} z_{s}\right)\right]^{2}}\right\},
\end{aligned}
$$

for the individual modes. From this equation we see that the first terms give a linear dependence of the phase, which only depends on the central frequency of the interfering pulses in the respective branches. The term between brackets, however, introduces a deviation from this linear behavior on the measured phase due to the GVD in the dispersive media. Note that almost all parameters in this term are known, as they can be derived from the measured amplitude as given by Eq. (3) by simply fitting a Gaussian to the pulse envelope. The individual $\beta_{s}$ are known for the different modes in the sample as a result of a Fourier transform. The term $\beta_{r} z_{r}$ is of course not exactly known, but drops out when comparing subsequent time frames due to the fact that the dispersion in the reference branch stays constant. However, the GVD, i.e., the $\beta^{\prime \prime}$ 's, are unknown in Eq. (7). Note that the measured amplitude for each mode directly gives a measure for $\kappa_{r}$ $+\kappa_{s}$ for that specific mode [see Eq. (3)]. This results from the fact that $\Gamma_{0}$ is a known constant. Subtraction of the obtained $\kappa_{r}+\kappa_{s}$ for the different modes therefore directly yields the relative difference $\left|\kappa_{s, T E 00}-\kappa_{s, T E 01}\right|$. From Eq. (2b) it can also be seen that this difference corresponds to $\left|\beta_{T E 00}^{\prime \prime}-\beta_{T E 01}^{\prime \prime}\right|$, which is a direct measure for the difference in GVD between the modes. Therefore we do know the relative difference between the $\beta^{\prime \prime}$ 's for the individual modes.

We can therefore depict the phase of the individual modes by plotting Eq. (7) using the experimentally determined parameters as a function of position on the sample. This is done in Fig. 9. At the same time the phase difference $\Delta \Phi$ between the modes as a function of position is plotted. Note that the phase of the individual modes shows only a very small deviation from linearity. Nevertheless, this deviation from linearity clearly shows up in the plotted phase difference between the modes (dotted line Fig. 9). By plotting the measured position of the phase singularities in this graph (indicated by crosses) it is exactly defined for which position the change of $\Delta \Phi$ should be equal to $\pi$. Note that the unknown $\beta_{r} z_{r}$ for the phase information only gives a linear

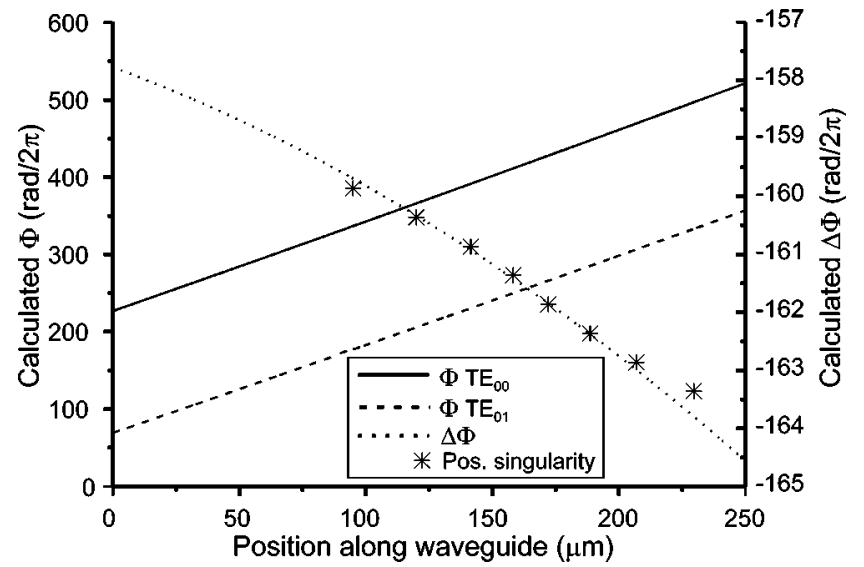

FIG. 9. (a) Calculated optical phase [Eq. (4)] for the two modes present in our measurement as a function of position on the waveguide. Experimental parameters used in this calculation are given in the text. The $\mathrm{TE}_{00}$ (solid line) and $\mathrm{TE}_{01}$ (dashed line) modes show an almost linear behavior. Deviation from linearity is however obvious when plotting the phase difference $\Delta \Phi$ between those two modes (dotted line). Note that between subsequent locations where singularities occur (indicated by crosses) the change for $\Delta \Phi$ has to be exactly equal to $\pi$. The shape of the $\Delta \Phi$ curve depends only on the $\beta^{\prime \prime} s$ for the individual modes. Therefore adjusting these parameters in the calculation so that the phase difference at the measured locations of the singularities is equal to $\pi$ allows a direct determination of the GVD for the individual modes.

offset in the phase and therefore does not affect the curvature. Thus it has no effect on the separation between the singularities. As the $\beta^{\prime \prime}$ 's are the only free parameters that can be adjusted to fit $\Delta \Phi$ to the phase differences obtained from the measured location of the phase singularities, it is clear that the GVD for individual modes can be retrieved from this measurement. Note that the linear offset has to be adjusted at the same time to define the location of the first singularity, but this does not affect the separation between the individual singularities as mentioned before.

Figure 9 qualitatively explains the nonconstant distance between singularities like the measured variation presented in Fig. 7. In Fig. 9 we can clearly see that the phase difference is nonlinear in position. If the slope of the $\Delta \Phi$ is steep, the phase changes rapidly and therefore the beat length becomes short. Based on our experimental observation as indicated by the crosses we expected to see a shallow slope at the left side (tail of the pulse) which becomes steeper towards the center of the image and then decreases again at the right side of the image (front of the pulse). However, in the calculation we do not observe the decreasing slope at larger positions/distances for the current experimental parameters. This is apparent from the deviation between the measured crosses and the theoretical curve. Setting slightly different values indicates that the model includes the observed behavior as is also apparent from Eq. (7). Note that the time dependence, i.e., as a function of $\tau$ as is measured in successive frames, is to first approximation linear in $\tau$ as can be seen in Eq. (7). As a result the $\Delta \Phi$, as depicted in Fig. 9, to first approximation shifts towards the right, linearly dependent on $\tau$. And this immediately shows how the beating length 
should change in Fig. 7 as a function of measurement frame. Note that the dependence of $\xi_{r}$ on $\tau$ introduces the deviation from this linear dependence as can be seen in Eq. (7).

In the current experimental data the accuracy for determining the amplitude of the $\mathrm{TE}_{01}$ is limited due to its relatively weak amplitude combined with the limited spectral resolution of the time sequences. As a result the accuracy for determining the GVD for the individual modes is limited as certain parameters have large error margins, which also explains the deviation between the expected and measured position of the phase singularities. It is, however, clear that in principle the demonstrated method allows the retrieval of the amplitude information of individual modes, provided that the modes are sufficiently separated in the Fourier domain. As a result we can directly measure the phase and group velocity for individual modes. From a comparison of the measured pulse length along the waveguide, the relative difference between the GVD for the modes is determined. The position of the phase singularities are a direct measure for the relative phase difference between the interfering modes. It is therefore clear that when both modes can be accurately retrieved from the measurement, it will be possible to retrieve the GVD for individual modes more accurately by the demonstrated method.

\section{CONCLUSION}

In conclusion, the propagation in time of a nearly Fourier limited laser pulse propagating through a bimodal waveguide has been visualized by an interferometric PSTM. The local amplitude and phase of the pulses has been retrieved so that both the phase and group velocity could be measured locally for both the $\mathrm{TE}_{00}$ and $\mathrm{TE}_{01}$ modes.

The measured optical amplitude shows a clear beating pattern as a function of position along the propagation direction as a result of the interference between the two modes in the waveguide. This beating pattern arises from the wavelength difference between both modes. We observe intriguing phase singularities arising from the interference. The distance between singularities is not constant and is even dependent on the reference time. This observation is a direct result of the different group velocity dispersion (GVD) experienced by the propagating modes. Extending an earlier analytical model to incorporate multi-mode propagation shows that it is possible to locally measure the GVD of the structure for individual modes. As a result, interesting and useful effects, such as pulse compression, pulse spreading, and pulse reshaping, now become accessible in local measurements. It is expected that the time resolved interferometric PSTM will in the near future be used for the local experimental investigation of physical phenomena inside (non)linear dispersive media, like integrated waveguide structures and photonic crystals.

\section{ACKNOWLEDGMENTS}

This research is part of the strategic Research Orientation on Advanced Photonic Structures of the MESA ${ }^{+}$Research Institute. Furthermore, this work is part of the research program of the Stichting voor Fundamenteel Onderzoek der Materie [FOM, financially supported by the Nederlandse Organisatie voor Wetenschappelijk Onderzoek (NWO)].
[1] M. Born and E. Wolf, Principles of Optics (Cambridge University Press, Cambridge, England, 1999), pp. 14-24.

[2] I. Walmsley, L. Waxer, and C. Dorrer, Rev. Sci. Instrum. 72, 1 (2001)

[3] J.C. Diels and R.W. Wolfgang, Ultrashort Laser Pulse Phenomena (Academic, San Diego, 1996).

[4] J.D. Joannopoulos, R.D. Meade, and J.N. Winn, Photonic Crystals: Molding the Flow of Light (Princeton University Press, Princeton, NJ, 1995).

[5] Photonic Crystals and Light Localization in the 21st Century, edited by C.M. Soukoulis, NATO Advanced Studies Institute C: Mathematical and Physical Sciences (Kluwer Academic, Dordrecht, The Netherlands, 2001), Vol. 563.

[6] S.G. Johnson, P.R. Villeneuve, S. Fan, and J.D. Joannopoulos, Phys. Rev. B 62, 8212 (2000).

[7] W. Nakagawa et al., J. Opt. Soc. Am. A 18, 1072 (2001).

[8] M. Scalora et al., Phys. Rev. A 52, 726 (1995).

[9] W. Chen and D.L. Mills, Phys. Rev. Lett. 58, 160 (1987).

[10] S. Yamada, Y. Watanabe, Y. Katayama, and J.B. Cole, J. Appl. Phys. 93, 1859 (2003).

[11] X. Letartre et al., Appl. Phys. Lett. 79, 2312 (2001).

[12] M. Notomi et al., Phys. Rev. Lett. 87, 253902 (2001).

[13] E. Schwoob et al., J. Opt. Soc. Am. B 19, 2403 (2002).
[14] M.C. Netti et al., Appl. Phys. Lett. 81, 3927 (2002).

[15] K. Inoue et al., Phys. Rev. B 65, 121308 (2002).

[16] For a classical example, see K. Smith and L.F. Mollenauer, Opt. Lett. 14, 1284 (1989).

[17] G.H. Vander Rhodes et al., IEEE J. Sel. Top. Quantum Electron. 6, 46 (2000).

[18] J.R. Krenn et al., Phys. Rev. Lett. 82, 2590 (1999).

[19] M.L.M. Balistreri et al., Opt. Lett. 24, 1829 (1999).

[20] P.L. Phillips et al., J. Appl. Phys. 85, 6337 (1999).

[21] C. Chicanne et al., Phys. Rev. Lett. 88, 097402 (2002).

[22] S.I. Bozhevolnyi et al., Phys. Rev. B 66, 235204 (2002).

[23] K. Okamoto et al., Appl. Phys. Lett. 82, 1676 (2003).

[24] M. Vaez-Iravani and R. Toledo-Crow, Appl. Phys. Lett. 62, 1044 (1992).

[25] M.L.M. Balistreri, J.P. Korterik, L. Kuipers, and N.F. van Hulst, Phys. Rev. Lett. 85, 294 (2000).

[26] E. Flück et al., J. Microsc. 202, 104 (2001).

[27] M.L.M. Balistreri, J.P. Korterik, L. Kuipers, and N.F. van Hulst, J. Lightwave Technol. 19, 1169 (2001).

[28] A. Nesci, R. Dändliker, M. Salt, and H.P. Herzig, Opt. Commun. 205, 229 (2002).

[29] M.L.M. Balistreri et al., Science 294, 1080 (2001).

[30] H. Gersen, J.P. Korterik, N.F. van Hulst, and L. Kuipers, Phys. 
Rev. E 68, 026604 (2003).

[31] Near-Field Optics, edited by D.W. Pohl and C. Courjon, NATO Advanced Studies Institute, Series E: Applied Sciences (Kluwer Academic, Dordrecht, The Netherlands, 1993), Vol. 242.
[32] J.R. Krenn, Nat. Mater. 2, 210 (2003).

[33] R.H.J. Kop and R. Sprik, Rev. Sci. Instrum. 66, 5459 (1995), and references therein.

[34] A. Imhof, W.L. Vos, R. Sprik, and A. Lagendijk, Phys. Rev. Lett. 83, 2942 (1999). 